Neutronen des Neutronengenerators bestrahlt ${ }^{8}$. Es wurde dann die erwartete weiche $\beta$-Strahlung mit einer Halbwertsdicke in Al von $60 \mu$ und mit großer Halbwertszeit beobachtet. Außerdem trat eine $\gamma$-Strahlung auf mit einer Absorbierbarkeit in $\mathrm{Pb}$ von $0,6 \mathrm{~cm}^{-1}$, die wahrscheinlich mit der oben gefundenen $\boldsymbol{\gamma}$-Strahlung von $1,42 \mathrm{MeV}$ iden- tisch ist. Die zur Bestätigung noch vorgesehenen Koinzidenzmessungen konnten allerdings nicht mehr vorgenommen werden, da die Versuche vorzeitig abgebrochen werden mußten.

8 Für die freundliche Überlassung des $\mathrm{HfO}_{2}$ bin ich Hrn. Prof. Dr. O. H a h n und Hrn. Dr. Fla m me r s f e l d zu Dank verpflichtet.

\title{
Über die Bedeutung schwerer Atome für die Verlangsamung von Neutronen
}

\author{
Von Karl Krebs \\ Aus dem Physikalischen Institut der Technischen Universität Berlin-Charlottenburg \\ (Z Naturforschg. 2 a, 435-438 [1947]; eingegangen am 6. April 1947)
}

Bei orientierenden Messungen über Dichteverteilung relativ langsamer (noch nicht thermischer) Neutronen von etwa $1 \mathrm{eV}$ wurde die Beobachtung gemacht, daß auch schwerere Atome - im wesentlichen handelte es sich um Kohlenstoff und Sauerstoff - in der Bremswirkung Wasserstoff ersetzen können, und zwar in einem höheren Maße als man von vornherein zu erwarten geneigt war ${ }^{1}$.

W Tegen der Bedeutung, die die Verlangsamung von Neutronen seinerzeit für verschiedene Probleme hatte, wurde diese Erscheinung in einer Anor.dnung, die Abb. 1 zeigt, genauer untersucht. Im Mittelpunkt einer radialsymmetrischen Anordnung befindet sich eine Neutronenquelle bekannter Art (Beryllium + Ra-Emanation), welche Neutronen von einigen $(5,6) \mathrm{MeV}$ emittiert. Diese ist kugelförmig von einem Hohlraum umgeben, in den verschiedene Substanzen eingefüllt werden können, deren verlangsamende Wirkung untersucht werden soll. Die ganze Versuchsanordnung befindet sich in einem mit Wasser gefüllten Gefäß. Ein Paraffinstopfen füllt das zur Einbringung des Neutronenpräparates dienende Rohr aus, um die Verhältnisse so gut wie möglich der Umgebung anzupassen. Im inneren Hohlraum - im gleichen wie die Neutronenquelle - befindet sich als Indikator eine Indiumfolie von etwa $1,5 \mathrm{~cm}^{2}$, und zwar in einer kleinen Cadmiumkassette, um die Wirkung rein thermischer Neutronen auszuschalten. Für Neutronen von etwa $1 \mathrm{eV}$ besitzt Indium bekanntlich ein Resonanzniveau, in dem es $\beta$-aktiv wird

1 Die Messungen wurden 1941 durchgeführt und mußten damals abgebrochen werden. Verf. hatte seitdem keine Möglichkeit mehr, die neuere einschlägige Literatur zu verfolgen, so daß die Ergebnisse vielleicht überholt sind; sie seien aber hier mitgeteilt, weil sie für den einen oder anderen der heute auf diesem Gebiet Arbeitenden doch von Interesse sein könnten.

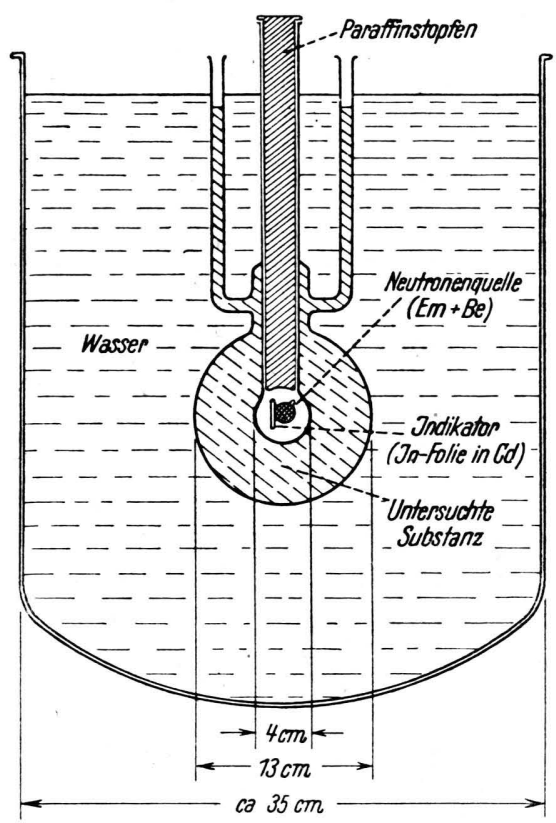

Abb. 1. Belichtungsanordnung.

- mit einer Halbwertszeit von 54 Min. -, so daß durch eine anschließende Zählrohrmessung bequem ein Maß für die Dichte von $1 \mathrm{eV}$-Neutronen zu erhalten ist. Die Benutzung des kleinen substanzfreien Hohlraumes hat den Vorteil, daß die Neutronendichte darin konstant und die Mes- 
sung von kleinen Lageänderungen des Indikators und der Neutronenquelle unabhängig ist.

Durch Füllung des Kugelraumes mit einer Reihe von verschiedenen Kohlenwasserstoffen wurden verschiedene Konzentrationen von Wasserstoff, Kohlenstoff und Sauerstoff hergestellt und darin die Indikatoren eine gewisse Zeit (im allgemeinen 1 Stunde) lang exponiert und anschließend ebenso lang ausgezählt. Die Zählung erfolgte mit einem kleinen Zählrohr von etwa $2 \times 4 \mathrm{~cm}^{2}$ Querschnitt und einer $0,1 \mathrm{~mm}$ starken, innen vergoldeten Dur-

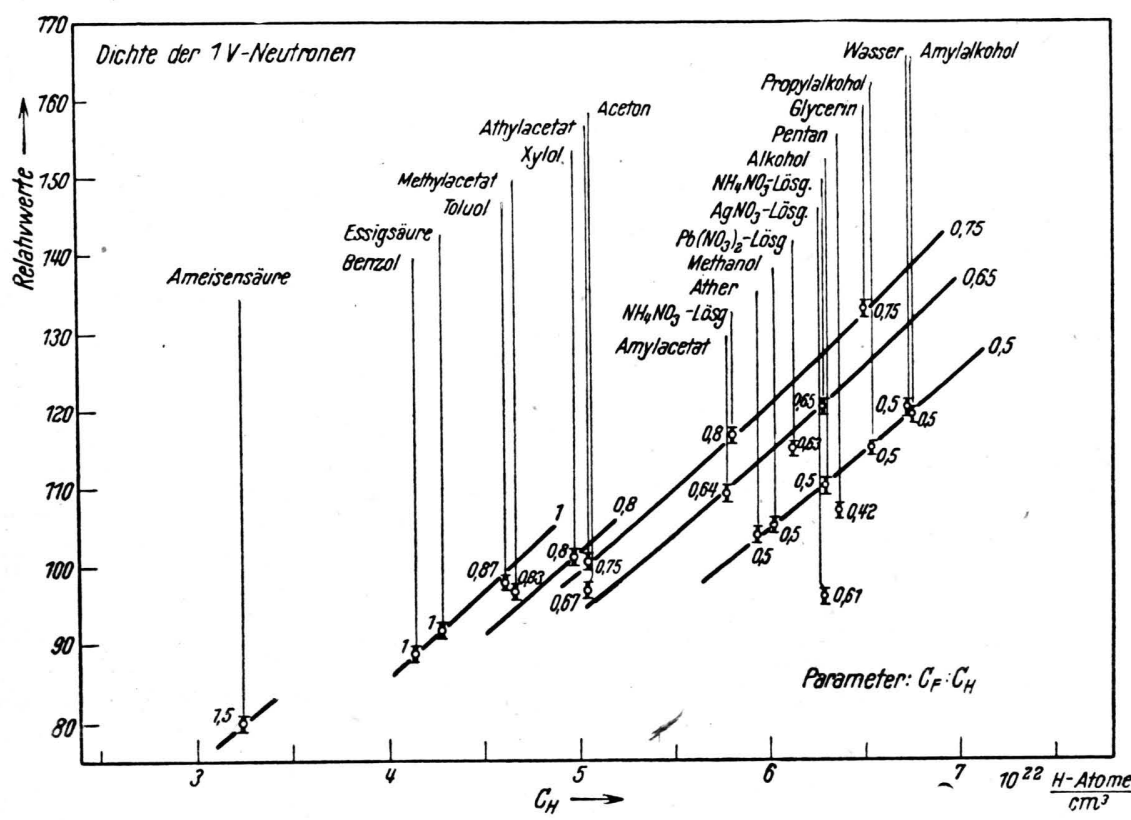

Abb. 2. Indium-Aktivität in Abhängigkeit von der H-Konzentration, mit äußerer Wasserfüllung.

aluminiumfolie als Wandung. Die üblichen Korrekturen wurden angebracht, so hinsichtlich des Auflösungsvermögens der Zählapparatur nach der Methode von Volz, Korrekturen auf Aktivitätsabfall des Neutronenpräparates, Nulleffekt des Zählrohrs usw. sowie auf gleiche Foliengröße, da zur Zeitersparnis mit mehreren Folien gearbeitet wurde, deren Größenverhältnis experimentell bestimmt worden war. Die gemessenen Teilchenzahlen lagen zwischen 5000 und 12000 , so daß der mittlere Fehler der Messungen etwa $1-2 \%$ beträgt. Die Dichten und damit die Atomkonzentrationen der verschiedenen untersuchten Substanzen können demgegenüber als praktisch fehlerlos betrachtet werden.

Die Ergebnisse dieser Meßreihen zeigt Abb. 2. Die Dichte der $1 \mathrm{eV}$-Neutronen wurde hier gegen die Wasserstoffkonzentration aufgetragen. Es ergab sich durchaus keine geschlossene Linie, sondern ein auf den ersten Blick recht unregelmäßiges Bild. Einer der Punkte (von Glycerin) lag sogar beträchtlich, etwa $10 \%$, über dem für Wasser erhaltenen Wert. Eine Ordnung unter all den verschiedenen Punkten wird erreicht, indem man das Konzentrationsverhältnis Fremdatome : Wasserstoffatome $\left(c_{\mathrm{F}}: c_{\mathrm{H}}\right)$ als Parameter benutzt (wobei mit Fremdatomen hier der Kürze halber Kohlenstoff- und Sauerstoffatome gemeint sind, zwischen denen in der vorliegenden Arbeit nicht besonders unterschieden wurde). Diese Werte $c_{\mathrm{F}} / c_{\mathrm{H}}$ sind in der Abb. 2 an den einzelnen Punkten mit angegeben. Die Werte von Substanzen mit gleichem $c_{\mathrm{F}} / c_{\mathrm{H}}$ liegen dann recht gut auf einander ähnlichen Kurven. Eine Reihe von Lösungen von Ammonium- und Bleinitrat, deren atomare $\mathrm{Zu}$ sammensetzung durch genaue Dichtebestimmung ermittelt wurde, ordnet sich ganz in dieses Schema ein. Eine Ausnahme bildet Silbernitrat, das ja wegen der bekannten Resonanzstellen des Silbers bei $2-3$ Volt eine Neutronenverarmung bei 1 Volt auch erwarten läßt. Die Abbildung zeigt, daß bei konstantem $c_{\mathrm{H}}$ eine Erhöhung von $c_{\mathrm{F}}$ auch eine deutliche Dichteerhöhung der $1 \mathrm{eV}$-Neutronen ergibt.

Dieser Effekt, nach dem es ziemlich gleichgültig ist, ob Wasserstoff- oder Fremdatome in der verlangsamenden Substanz benutzt werden, fällt besonders auf, wenn man dieselben Ergebnisse als Funktion der Gesamtkonzentration Atome $/ \mathrm{cm}^{3}$ aufträgt (siehe Abb.3). Auch das Herausfallen der Silbernitratlösung wird hier besonders deutlich.

Wie ist dieser Befund nun zu erklären? Offenbar so, daß die Fremdatome zwar nicht - oder nur wenig - verlangsamend wirken, aber doch nach Maßgabe ihrer Konzentration die Abdiffusion schneller Neutronen, aus denen die langsamen ja letzten Endes entstehen, verhindern.

Ist diese Erklärung richtig, dann muf in der Peripherie der untersuchten Füllungen die Dichte 
schneller Neutronen mit wachsendem $c_{\mathrm{F}} / c_{\mathrm{H}}$ abnehmen. Dies konnte auf folgende Weise - wenigstens qualitativ bestätigt werden: Es wurden dieselben Messungen an der Apparatur der Abb. 1, und zwar ohne Wasserfüllung des äußeren Gefäßes, wiederholt, wobei ohne wesentlich neue Ergebnisse. eine ganz der Abb. 2 entsprechende Meßreihe entstand, die nur wegen des Wegfallens der Rückdiffusion aus dem Wasserbassin etwas tiefer liegt. Die Differenz der beiden Meßreihen muß, wie man leicht einsieht, ein qualitatives Maß sein für die die Peripherie nach außen durchsetzenden schnellen

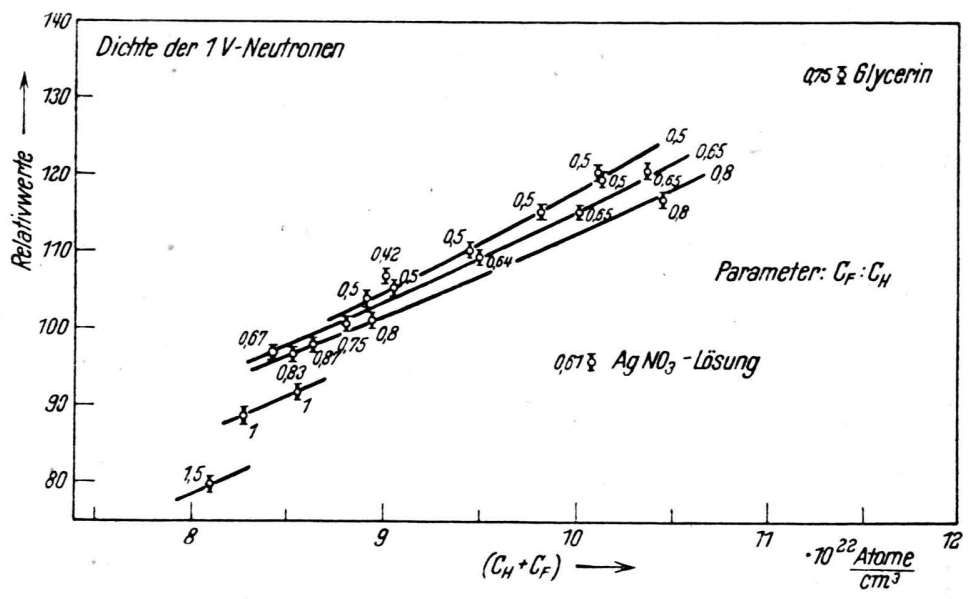
Neutronen, also auch für deren dortige

Abb. 3. Indium-Aktivität in Abhängigkeit von der Gesamtkonzentration, mit äußerer Wasserfüllung.

Dichte. In der Abb. 4 sind die beiden Meßreihen zusammengestellt und ihre Differenz eingezeichnet. Daß diese Differenzen ganz allgemein mit steigender Konzentration abnehmen, ist von vornherein zu erwarten. Man erkennt jedoch noch darüber

steigender Konzentration der Fremdatome abnimmt.

In der Abb. 5 sind die Ergebnisse der Messungen ohne Wasserfüllung des Gefäßes noch einmal in anderer Weise zusammengestellt, derart, daß die

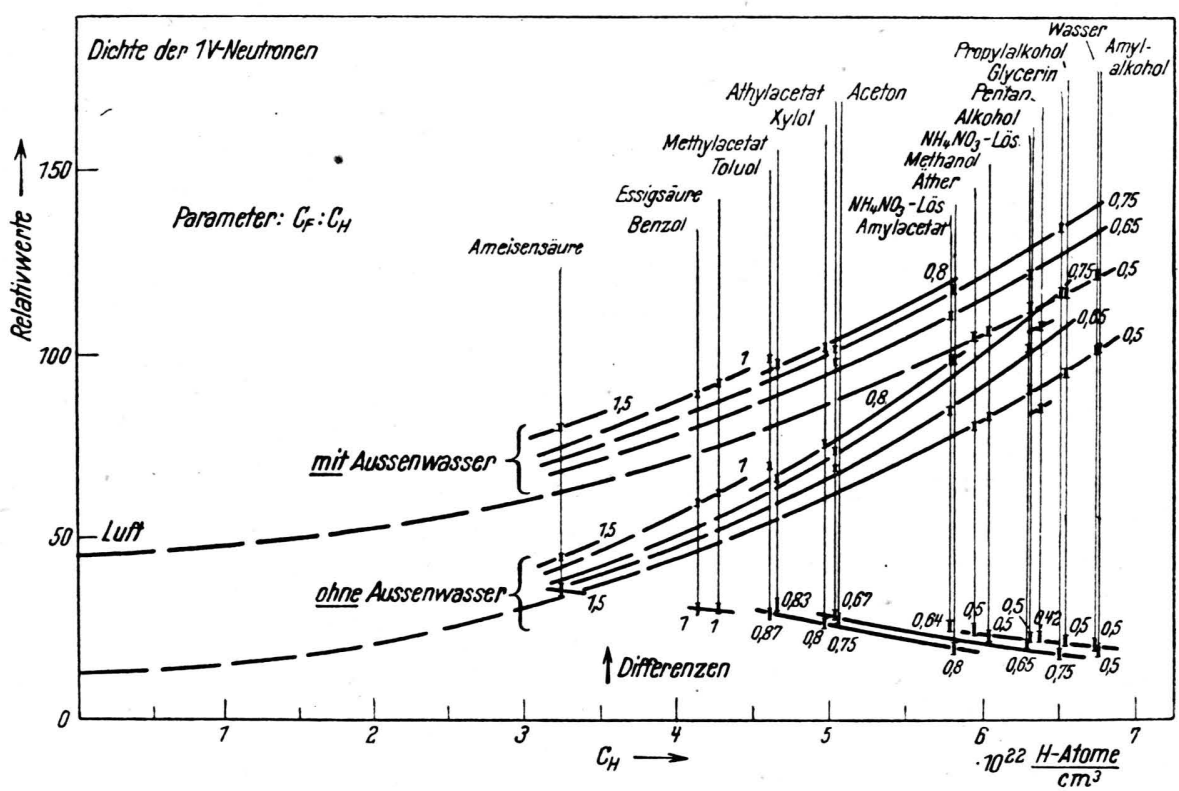

Abb.4. Zusammenstellung der Meßreihen mit und ohne äußere Wasserfüllung.

(Die Schnittpunkte der beiden Kurven mit der Ordinatenachse sind je durch einen ' versehentlich nicht eingezeichneten Meßpunkt gesichert.)

hinaus, daß die Differenz bei konstanter Wasserstoffkonzentration mit steigendem $c_{\mathrm{F}} / c_{\mathrm{H}}$, dessen Wert wieder an den einzelnen Punkten angegeben ist, abnimmt, daß also nach dem oben Gesagten die Dichte schneller Neutronen an der Peripherie mit
Gesamtkonzentration aufgetragen ist und als Parameter der prozentuale Wasserstoffgehalt. Zur Untersuchung des gaskinetischen Verhaltens der schnellen Neutronen wäre diese Darstellung einer näheren, rechnerischen Behandlung sicher am 
besten zugänglich, worauf im Rahmen dieses Kurzberichtes jedoch nur hingewiesen sei.

Eine weitere Konsequenz aus der Dichteerhöhung der $1 \mathrm{eV}$-Neutronen im Mittelpunkt bei steigendem $c_{\mathrm{F}} / c_{\mathrm{H}}$ ergibt sich aus folgender Überlegung: Die Gesamtzahl aller $1 \mathrm{eV}$-Neutronen muß bei genügend großen (und hier auch vorliegenden) Dimensionen des äußeren Gefäßes unabhängig von der Kugelfüllung dieselbe bleiben. Ist bei einer bestimmten Füllung die Dichte im Mittelpunkt grö-
Es ist erkennbar, daß der Abfall für Glycerin steiler verläuft als für Wasser, wenn auch dieser Effekt nicht sehr ausgeprägt ist und nur wenig außerhalb der Fehlergrenzen liegt. (Glycerin und Propylalkohol wären für diesen Vergleich geeigneter gewesen, da sie beide praktisch gleichen Wasserstoffgehalt und nur verschiedene Konzentrationen an Fremdatomen aufweisen; doch konnten diese Messungen nicht mehr zur Durchführung kommen.)

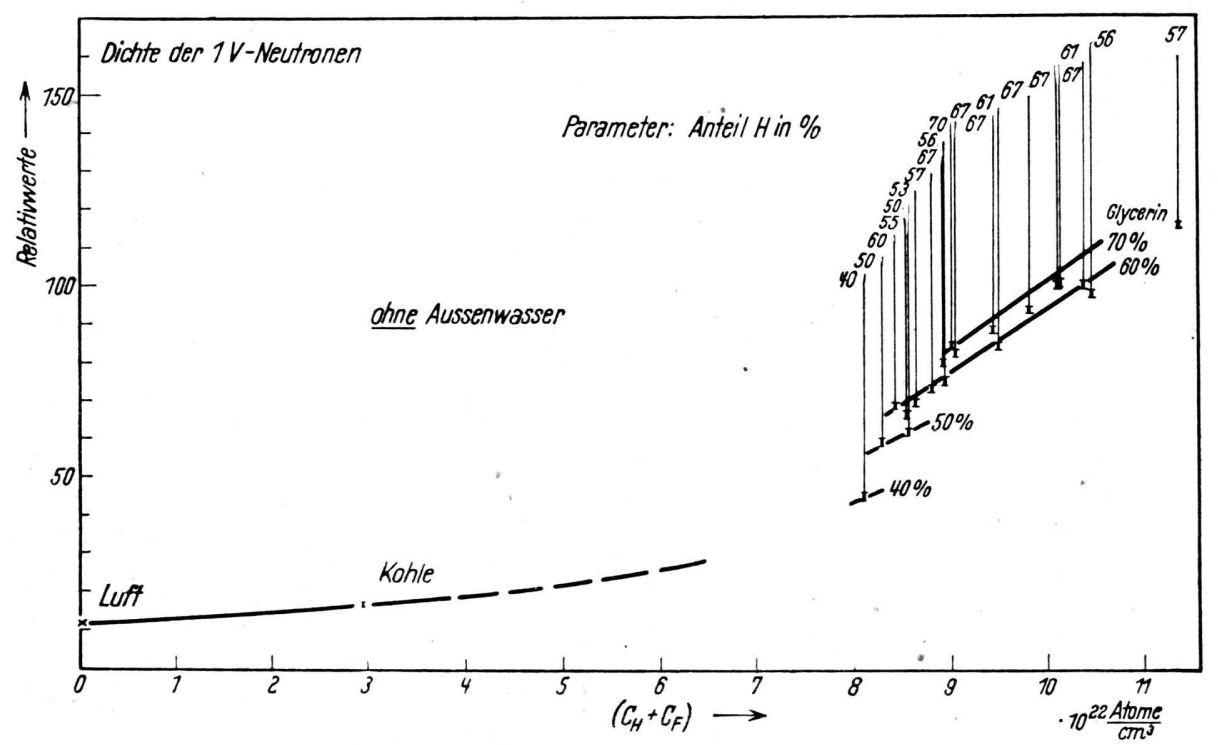

Abb. 5. Indium-Aktivität in Abhängigkeit von der Gesamtkonzentration, ohne äußere Wasserfüllung.

ßer, so muß sie nach außen hin entsprechend kleiner, die „Verteilungskurve“ also steiler sein. Durch Vergleichsmessungen an der Peripherie der äußeren Kugel und im Innenhohlraum wurden für Wasser und Glycerin je zwei Punkte der Verteilungskurve bestimmt. Das Ergebnis zeigt Tab.1, wobei die Werte im Innenraum $=100$ gesetzt und die Fehlergrenzen der anderen Werte entsprechend erweitert wurden:

\begin{tabular}{|c|c|c|}
\hline & Innenhohlraum & $\begin{array}{c}\text { Peripherie } \\
r=6,5 \mathrm{~cm}\end{array}$ \\
\hline Wasser & 100 & $\mathbf{5 0 , 8 \pm 0 , 8}$ \\
Glycerin & 100 & $48,6 \pm 0,8$ \\
\hline
\end{tabular}

Tab. 1.
Zum Schluß sei noch kurz angedeutet, weshalb dem Einfluß der Fremdatome eine solche Beachtung geschenkt wurde. Der an sich nicht übermäßige Effekt der Dichteerhöhung langsamer Neutronen bei Mitverwendung schwererer Atome in der Bremssubstanz schien seinerzeit von großer Bedeutung, weil man auf diese Weise bei Inkaufnahme gleicher Neutronenverluste die Dimensionen gewisser Untersuchungsapparaturen hätte kleiner halten können. Auch schien die Verwendung von Glycerin oder auch schweren ölen (die nach neueren Erkenntnissen allerdings Deuterium statt Wasserstoff enthalten müßten) noch aus einem anderen Grunde der Untersuchung wert: Bei einer eventuellen Ausnutzung der in der Bremssubstanz erzeugten Wärme bestünde wegen der höheren Siedetemperatur dieser Substanzen die Möglichkeit, beim Betrieb irgendwelcher Wärmekraftmaschinen höhere thermodynamische Wirkungsgrade $\mathrm{zu}$ erzielen, als sie selbst mit überhitztem Wasserdampf möglich wären. 